This item was submitted to Loughborough's Research Repository by the author.

Items in Figshare are protected by copyright, with all rights reserved, unless otherwise indicated.

\title{
Generation of low-frequency rayleigh waves by heavy lorries
}

\section{PLEASE CITE THE PUBLISHED VERSION}

http://www.multi-science.co.uk/lowfreq.htm

\section{PUBLISHER}

(C) Multi-Science Publishing

\section{VERSION}

VoR (Version of Record)

\section{LICENCE}

CC BY-NC-ND 4.0

\section{REPOSITORY RECORD}

Krylov, Victor V.. 2012. "Generation of Low-frequency Rayleigh Waves by Heavy Lorries". figshare. https://hdl.handle.net/2134/10061. 
This item was submitted to Loughborough's Institutional Repository (https://dspace.lboro.ac.uk/) by the author and is made available under the following Creative Commons Licence conditions.

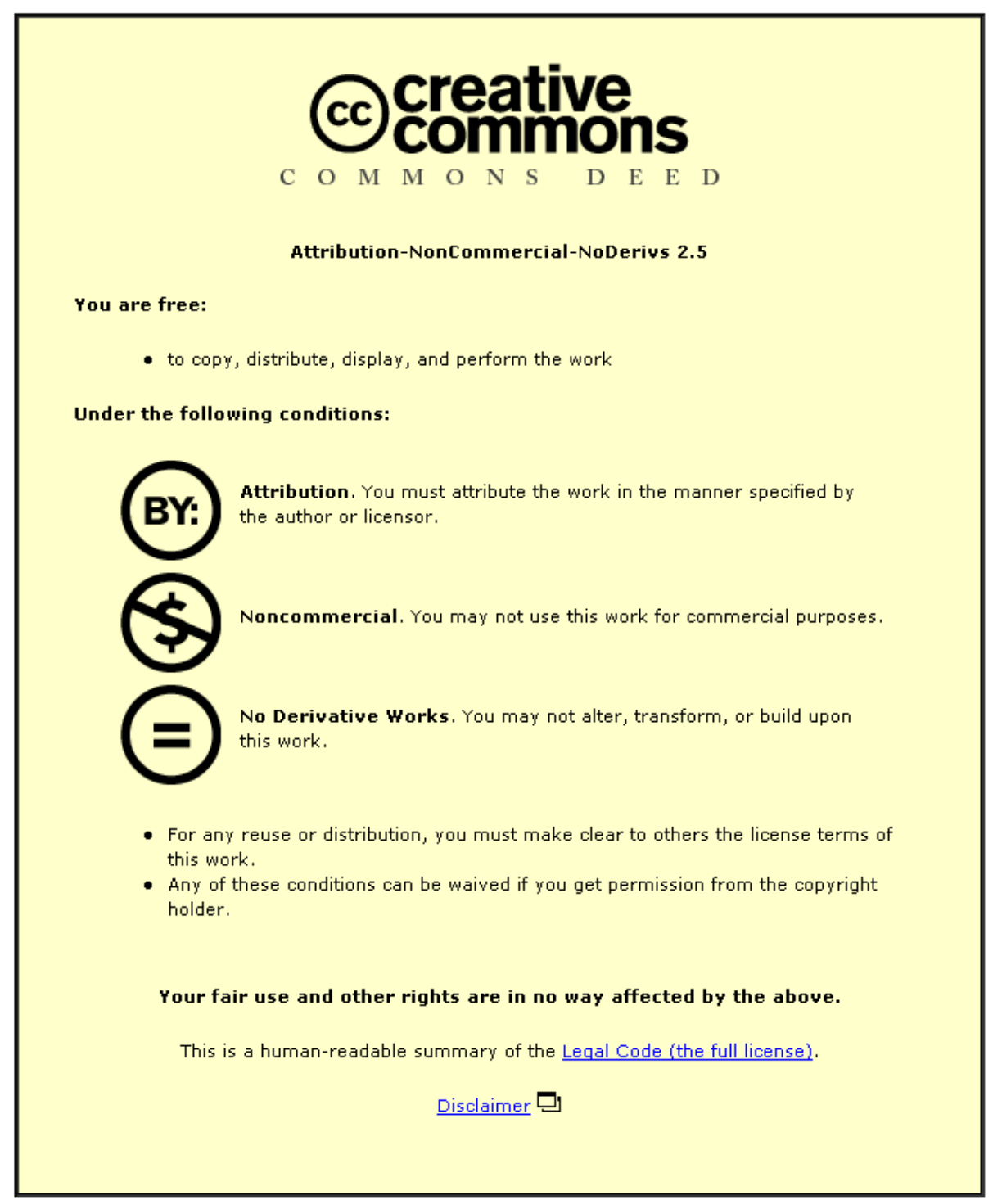

For the full text of this licence, please go to: http://creativecommons.org/licenses/by-nc-nd/2.5/ 
Generation of Low-Frequency Rayleigh Waves by Heavy Lorries

\author{
by \\ V.V. Krylov \\ Reprinted from \\ JOURNAL OF \\ LOW FREQUENCY \\ NOISE \& VIBRATION
}

VOLUME 14 No. 41995

MULTI-SCIENCE PUBLISHING CO. LTD.

107 High Street, Brentwood, Essex CM14 4RX, United Kingdom 


\title{
Generation of Low-Frequency Rayleigh Waves by Heavy Lorries
}

\author{
V.V. Krylov \\ Centre for Research into the Built Environment, Nottingham Trent University, \\ Burton Street, Nottingham NG1 4BU, UK
}

Received 25 February, 1995

\begin{abstract}
Generation of low-frequency ground vibrations by heavy lorries is considered theoretically for both vehicles accelerating (decelerating) with a constant acceleration and vehicles travelling at constant speed on damaged or bumpy surfaces. In the case of damaged or bumpy surfaces, excitation of axle-hop resonances is taken into account, whereas an accelerating or braking vehicle is modelled as a point horizontal traction force applied to the ground and moving along with the vehicle. Frequency spectra of the vertical component of the ground vibration velocity are investigated for different functions of road surface roughness, acceleration, final (initial) speed of the vehicle, ground attenuation, Poisson's ratio, and radiation angle relative to the direction of the vehicle movement. It is shown that damaged or bumpy road surfaces normally generate vibrations of higher amplitudes, in comparison with accelerating and braking lorries. In contrast to vehicles travelling along bumpy or uneven roads and generating vibrations propagating at all directions, the ground vibrations generated by accelerating and braking vehicles are characterised by the directivity function showing that there is no radiation in the direction perpendicular to the vehicle movement.
\end{abstract}

\section{INTRODUCTION}

Ground vibrations from road vehicles, especially from heavy lorries, may result in significant nuisance to residents living in the nearby properties and cause fear of possible damage to the dwellings. According to the survey of vibration nuisance from road traffic ${ }^{1}$, among the most annoying mechanisms of trafficinduced ground vibrations are: 1) vehicles accelerating and braking respectively $49.7 \%$ and $30.2 \%$ of the respondents, and 2) damaged or bumpy road surfaces $-39.4 \%$ of the respondents. In this paper both these main mechanisms of generating ground vibrations are investigated theoretically.

We consider vehicles travelling at constant speed on damaged or bumpy surfaces, and vehicles accelerating (decelerating) with a constant acceleration $\alpha$ from rest to a constant speed, or braking from a constant speed to a stop. In the case of uneven road surfaces excitation of the most important vehicle resonances is taken into account. However, an accelerating or braking vehicle of mass $\mathrm{M}$ is modelled as a point horizontal traction force $\mathrm{F}_{\mathrm{x}}=\alpha \mathrm{M}$ applied to the ground and moving along with the vehicle. Frequency spectra of the vertical component of the ground vibration velocity are investigated using the Green's function method for different functions of road surface roughness, acceleration, final (initial) speed of the vehicle, ground attenuation, Poisson's ratio, and 
radiation angle $\Theta$ relative to the direction of the vehicle movement.

While ground vibrations due to uneven road surfaces were investigated both analytically and numerically by several authors, there is no adequate theoretical research where vibrations from accelerating or braking vehicles were considered. This is why the main attention in this paper is paid to generating ground vibrations by accelerating and braking lorries.

The existing literature on elastic wave radiation by forces moving at nonconstant trans-Rayleigh speeds along a free surface of an elastic half space ${ }^{2}$ is not directly applicable to the case of road-traffic-induced ground vibrations where vehicle speeds are invariably much lower than Rayleigh wave velocities. Note in this connection, that trans-Rayleigh train speeds are a reality and have important implications for the generation of ground vibrations ${ }^{3}$.

It should be emphasised that the main mechanism of ground vibration generation by accelerating or decelerating vehicles is the action of horizontal traction forces which are applied from tyres to the ground only during the time of accelerating or braking (the contribution of normal load forces is relatively small for speeds and accelerations typical for road traffic). However, for vehicles travelling along uneven or bumpy roads, the most important are vertical dynamic forces caused by the obstacles.

\section{ACCELERATING AND BRAKING LORRIES}

In this section we briefly describe the main features of generating ground vibrations by accelerating and braking lorries and discuss some numerical results.

Being interested only in low-frequency ground vibrations typical of trafficinduced mechanisms of generation and assuming that the main vehicle resonances are not excited during horizontal movement, we model an accelerating or braking vehicle as a point load travelling along the surface either with acceleration or deceleration (Figure 1). The low-frequency approximation assumes that the shortest wave-lengths of the generated ground vibration spectra are larger than the dimensions of the vehicle.

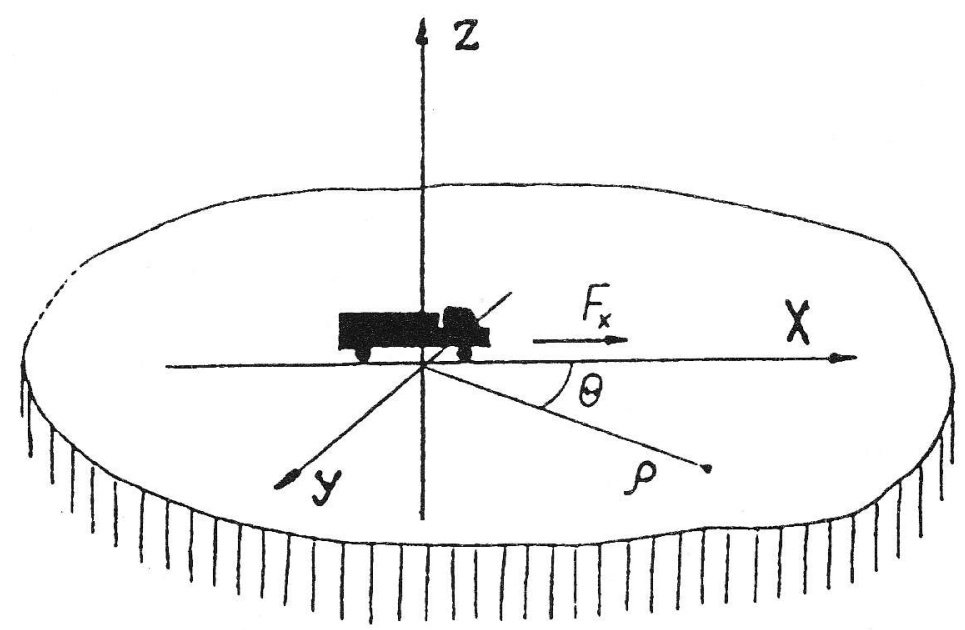

Figure 1. Geometry of the problem.

If a vehicle is accelerated or decelerated at constant absolute value $\alpha$, then the point load force applied from a vehicle to the ground surface is a horizontal traction force that moves along the $\mathrm{x}$-axis with a vehicle and has the amplitude 


$$
\mathrm{F}_{\mathrm{x}}=\alpha \mathrm{M}
$$

The related mechanical shear stresses applied to the ground surface during acceleration or deceleration, i.e. during the period of time from $t=0$ to $t=v / \alpha$, where $v$ is the final or initial speed, are described respectively as follows:

$$
\mathrm{T}_{\mathrm{xz}}(\rho, \mathrm{t})=-\mathrm{F}_{\mathrm{x}} \delta\left(\mathrm{x}-\alpha \mathrm{t}^{2} / 2\right) \delta(\mathrm{y})
$$

and

$$
T_{x z}(\rho, t)=F_{x} \delta\left(x-v t+\alpha t^{2} / 2\right) \delta(y)
$$

Here $T_{i j}$, where $i, j=1,2,3$, are the components of a load stress tensor applied to the surface, $\rho=\{x, y\}$ is the surface radius-vector, and $\delta(z)$ is Dirac's deltafunction.

The ground vibration field generated by accelerating or braking vehicles in an elastic half space, which we assume to be homogeneous and isotropic, should satisfy the elastic Lamé equation

$$
(\lambda+2 \mu) \operatorname{grad} \operatorname{div} u-\mu \operatorname{rot} \operatorname{rot} u-\rho_{0} \partial 2 u / \partial t^{2}=0
$$

and the boundary conditions on the ground surface taking into account only horizontal traction forces (2) and (3):

$$
\begin{gathered}
\sigma_{x z}=2 \mu u_{x z}=-T_{x z}(\rho, t) \\
\sigma_{y z}=2 \mu u_{y z}=0 \\
\sigma_{z z}=\lambda u_{n m}+2 \mu u_{z z}=0
\end{gathered}
$$

Here $\mathrm{u}$ is the particle displacement vector with the components $u_{i} ; \lambda$ and $\mu$ are the elastic Lamé constants; and $\mathrm{u}_{\mathrm{ij}}=(1 / 2)\left(\partial \mathrm{u}_{\mathrm{i}} / \partial \mathrm{x}_{\mathrm{j}}+\partial \mathrm{u}_{\mathrm{j}} / \partial \mathrm{x}_{\mathrm{j}}\right)$ are the components of the linearised deformation tensor. Without loss of generality, we will limit our calculation of frequency spectra to the vertical component of ground vibration velocity $\mathrm{v}_{\mathrm{z}}=\mathrm{du}_{\mathrm{z}} / \mathrm{dt}$ which is usually measured in experimental observations.

The solution to the problem (1)-(5) using the Green's function method and taking into account only the contribution of generated Rayleigh surface waves results in the following expression for the vertical component of the surface velocity spectrum:

$$
\begin{aligned}
v_{z}(\rho, \Theta, \omega)= & \left(\frac{2 \pi}{k_{R} \rho}\right)^{1 / 2} \frac{\omega k_{R}^{2}\left[k_{t}^{2} v_{1} v_{t}-k_{R}^{2} b\left(k_{R}\right)\right] \cos \Theta}{\pi \mu F^{\prime}\left(k_{R}\right) k_{t}^{2}} \times \\
& T_{x z}\left(\omega, k_{R} \cos \Theta\right) e^{-k_{R} \gamma \rho} e^{i k_{R} \rho-i 3 \pi / 4}
\end{aligned}
$$

Here the following notations are used: $\rho=\rho(x, y)$ is the distance to the observation point; $k_{R}=\omega / c_{R}$ is the Rayleigh wave number, where $c_{R}$ is the Rayleigh wave velocity; $b\left(k_{R}\right)=2 k_{R}^{2}-k_{t}^{2}-2 v_{1} v_{t}, v_{1, t}=\left(k_{R}^{2}-k_{1, t}^{2}\right)^{1 / 2}$ are nonspecified expressions, where $k_{1, t}=\omega / c_{1, t}$ are the wavenumbers of bulk longitudinal and shear acoustic waves, $c_{1}$ and $c_{t}$ are their phase velocities; $F^{\prime}\left(k_{R}\right)$ is the derivative $\mathrm{dF}(\mathrm{k}) / \mathrm{dk}$ of the Rayleigh determinant $\mathrm{F}(\mathrm{k})=\left(2 \mathrm{k}^{2}-\mathrm{k}_{\mathrm{t}}^{2}\right)-4 \mathrm{k}^{2} \mathrm{v}_{1} \mathrm{v}_{\mathrm{t}}$ taken at $\mathrm{k}=\mathrm{k}_{\mathrm{R}} ; \Theta=\cos ^{-1}(\mathrm{x} / \rho)$ is the observation angle; and $\mathrm{T}_{\mathrm{xz}}\left(\omega, \mathrm{k}_{\mathrm{R}} \cos \theta\right)$ is 
the Fourier transform of the load force (eqns (2) or (3)) which has the following expressions for accelerating and braking vehicles respectively:

$$
\begin{gathered}
\mathrm{T}_{\mathrm{xz}}\left(\omega, \mathrm{k}_{\mathrm{R}} \cos \theta\right)=-(1 / 2 \pi) \alpha \mathrm{M} \int_{0}^{\mathrm{v} / \mathrm{a}} \mathrm{e}^{\mathrm{i} \omega t-\mathrm{ik} \mathrm{k}_{\mathrm{R}} \cos \Theta\left(\mathrm{at}^{2} / 2\right)} \mathrm{dt} \\
\mathrm{T}_{\mathrm{xz}}\left(\omega, \mathrm{k}_{\mathrm{R}} \cos \theta\right)=-(1 / 2 \pi) \alpha \mathrm{M} \int_{0}^{\mathrm{v} / \mathrm{a}} \mathrm{e}^{\mathrm{i}\left(\omega t-\mathrm{k}_{\mathrm{R}} v \cos \Theta\right) t+\mathrm{ti} \mathrm{k}_{\mathrm{R}} \cos \Theta\left(\mathrm{at}^{2} / 2\right)} \mathrm{dt}
\end{gathered}
$$

In writing (6) we have taken account of attenuation of generated ground vibrations in the ground by replacing the wavenumber of a Rayleigh wave in an ideal elastic medium $k_{R}=\omega / c_{R}$ by the complex wavenumber $k_{R}{ }^{\prime}=k_{R}(1+i \gamma)=$ $\left(\omega / c_{R}\right)(1+i \gamma)$. Here $\gamma \ll 1$ is a positive constant which describes the linear dependence of a Rayleigh wave attenuation coefficient on frequency $\omega$. For different types of ground $\gamma$ are in the range from 0.01 to $0.2 .{ }^{4}$

One can prove that (7) and (8), corresponding to accelerating and braking vehicles respectively, differ from each other only by the phase factor. Therefore, being interested only in amplitudes of ground vibrations $V(\rho, \omega)=\left|v_{z}(\rho, \omega)\right|$, in what follows we describe the $1 / 3$-octave ground vibration spectra generated only by braking vehicles. We use both direct numerical calculations of (6)-(8) and calculations based on the simple analytical approximation of (7) and (8) neglecting the exact position of the vehicle during acceleration or deceleration. One can show that the above mentioned simple analytical approximation gives the same results for $1 / 3$-octave spectra as the approach based on the direct numerical calculations.

Figure 2 illustrates the behaviour of $1 / 3$-octave spectra of ground vibrations (in $\mathrm{dB}$ relative to the reference level of $10^{-9} \mathrm{~m} / \mathrm{s}$ ) generated by a braking lorry with $\mathrm{M}=20000 \mathrm{~kg}$ for three different values of deceleration: $\alpha=1,5$ and $9 \mathrm{~m} / \mathrm{s}^{2}$ (curves 1,2 , and 3 respectively). The initial speed is $v=10 \mathrm{~m} / \mathrm{s}$. Other parameters are the following: mass density of the ground $\rho_{0}$ is $2000 \mathrm{~kg} / \mathrm{m}^{3}$, velocity of longitudinal bulk waves $-c_{1}=471 \mathrm{~m} / \mathrm{s}$, shear waves $-c_{t}=272 \mathrm{~m} / \mathrm{s}$ and Rayleigh surface waves $-250 \mathrm{~m} / \mathrm{s}$ (this corresponds to a Poisson ratio of $\sigma$ $=0.25$ ). The constant of ground attenuation of Rayleigh waves was set as $\gamma=$ 0.05. It follows from Figure 2 that amplitudes of generated ground vibrations at all frequencies increase with increase of $\alpha$.

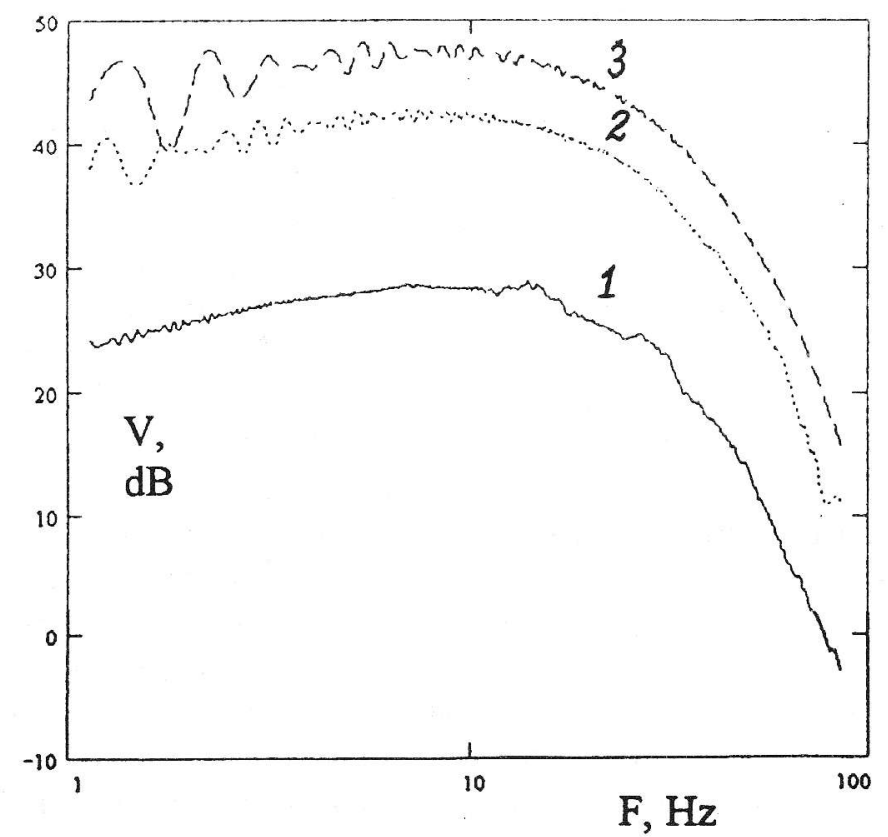

Figure 2. 1/3-octave spectra for different accelerations. $1=1 \mathrm{~m} / \mathrm{s}^{2} ; 2=5$ $\mathrm{m} / \mathrm{s}^{2} ; 3=9 \mathrm{~m} / \mathrm{s}^{2}$. 
The behaviour of generated ground vibration spectra for three different values of the initial speed $v=5,10$ and $20 \mathrm{~m} / \mathrm{s}$ (curves 1,2 , and 3 respectively) is shown on Figure 3 for $\alpha=5 \mathrm{~m} / \mathrm{s}^{2}$ and $\Theta=\pi / 3$. It is seen that for frequencies higher than $4-5 \mathrm{~Hz}$ the spectra are almost independent of $v$.

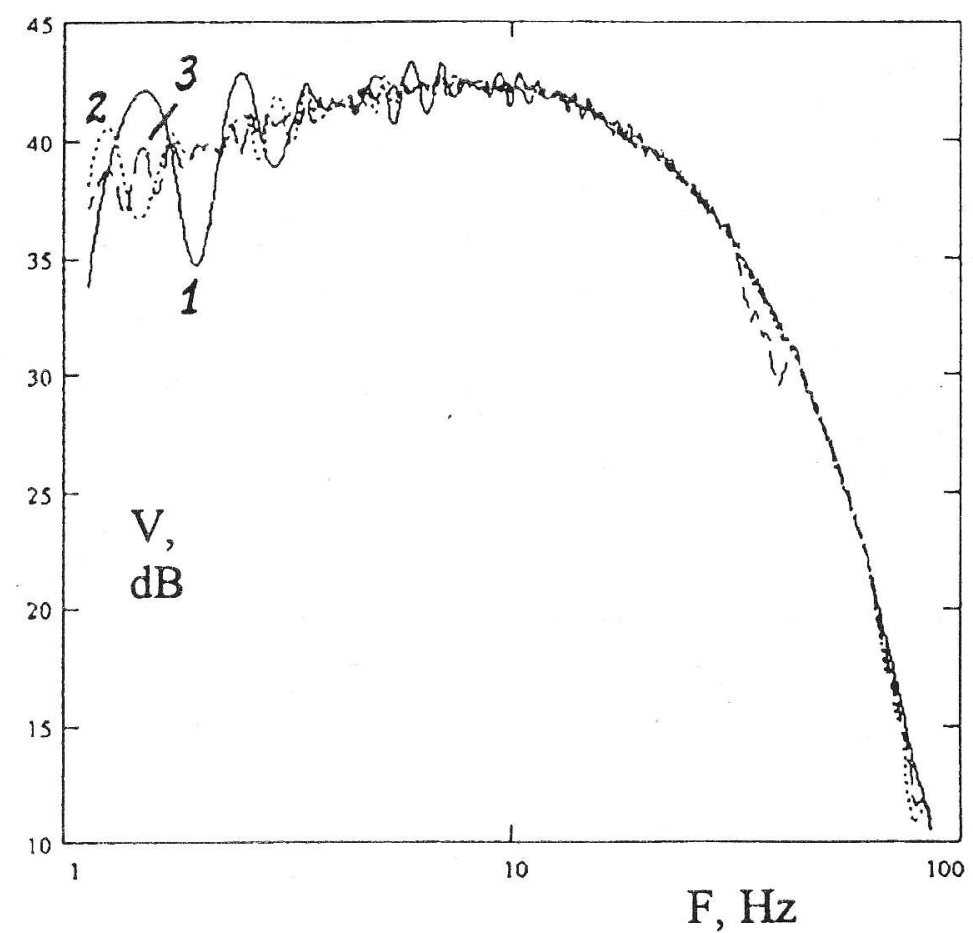

Figure 3. 1/3-octave spectra for different initial speeds. $1=5 \mathrm{~m} / \mathrm{s} ; 2=10$ $\mathrm{m} / \mathrm{s} ; 3=30 \mathrm{~m} / \mathrm{s}$.

In contrast to the case of generating ground vibrations by vehicles travelling on uneven roads, for which the load forces are directed normally to the ground surface, the accelerating and braking vehicles generate ground vibrations dependent on the observation angle $\Theta$ with respect to the vehicle movement. The directivity patterns of ground vibrations from accelerating or braking vehicles averaged over 1/3-octave frequency band are described by the function $\cos \Theta$ showing that there is no radiation at the angles $\Theta=\pi / 2$ and $3 \pi / 2$, i.e., in the directions perpendicular to the vehicle movement.

\section{LORRIES TRAVELLING ON DAMAGED OR BUMPY ROAD SURFACES}

As was mentioned before, theoretical investigations of generating ground vibrations by vehicles travelling on uneven surfaces have been carried out by several authors (see, e.g., Ref. 5). However, the attention in these investigations has been paid to statistically rough surfaces of rather good quality, whereas in many practical situations roads have localised uneven areas, such as pits resulting from surface damage or artificial bumps aiming to calm road traffic in some residential areas. Practical observations show that such pits and bumps may become sources of noticeable ground vibrations. In what follows we give a very brief description of the analysis of ground vibration generation by lorries travelling over pits or bumps.

A typical mechanical model of a road vehicle travelling on an uneven road possesses four degrees of freedom corresponding to four main resonance 
frequencies of low-frequency vibrations corresponding to body bounce and pitch, or to front- and rear-axle hops. Frequencies of body bounce and pitch resonances are normally very low (in the range of 1-3 Hz). Axle-hop resonance frequencies are essentially larger (from $8 \mathrm{~Hz}$ to $12 \mathrm{~Hz}$ ) and are therefore more important from the point of view of generating ground vibrations (we remind the reader that Rayleigh wave generation efficiency is higher at higher frequencies).

Keeping this in mind, we use a simplified model of a vehicle considering the carriage as immobile in vertical direction and taking into account only axle vibrations. This model consists of two identical vibrating systems each having one degree of freedom and comprising of an axle mass $\mathrm{m}$ and two springs with constants $\mathrm{K}_{1}$ and $\mathrm{K}_{2}$ modelling respectively the elasticities of tyre and suspension (Figure 4). It is taken into account that axles are separated from each other by the distance L. We also assume that pit or bump cross-section in the plane $y=0$ is described by the function $z_{1}=f(x)$.

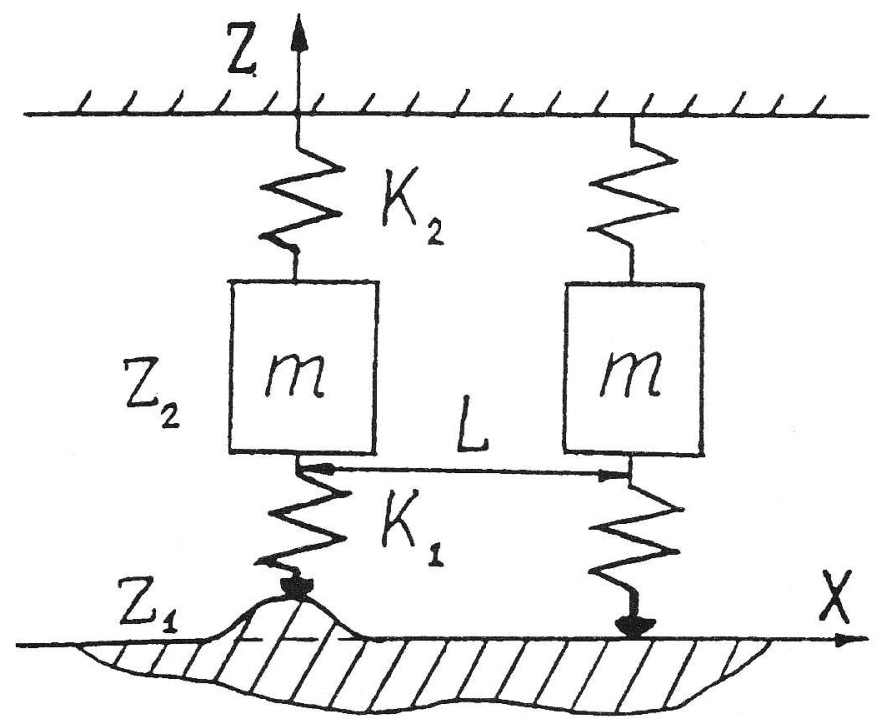

Figure 4. Mechanical model of a vehicle considering axle-hop resonances.

According to the model considered, the equation describing vertical displacements of each axle versus its static position $z_{2}$ has the form

$$
m \partial^{2} z_{2} / \partial t^{2}+Q \partial z_{2} / \partial t+K z_{2}=K_{1} z_{1}(v t)
$$

where $\mathrm{K}=\mathrm{K}_{1}+\mathrm{K}_{2}$ is a combined elasticity of tyre and suspension, and Q is a total damping coefficient. Assuming that damaged area of the road is small and its centre is located at $\mathrm{x}=0$ and $\mathrm{y}=0$, the related normal stress $\mathrm{T}_{\mathrm{zz}}$ applied from the vehicle to the ground may be written in the form

$$
\mathrm{T}_{z z}(\rho, \mathrm{t})=\mathrm{K}_{1}\left[\left(\mathrm{z}_{2}(\mathrm{t})-\mathrm{Z}_{1}(\mathrm{t})+\mathrm{z}_{2}(\mathrm{t}-\mathrm{L} / \mathrm{v})-\mathrm{Z}_{1}(\mathrm{t}-\mathrm{L} / \mathrm{v})\right] \delta(\mathrm{x}) \delta(\mathrm{y})\right.
$$

where $\mathrm{Z}_{1}(\mathrm{t})=\mathrm{z}_{1}(\mathrm{vt})$ and $\mathrm{Z}_{1}(\mathrm{t}-\mathrm{L} / \mathrm{v})=\mathrm{z}_{1}(\mathrm{vt}-\mathrm{L})$ are the input functions for the front and rear axles respectively.

As before, the generated ground vibrations should satisfy the dynamic Lamé equation (4). But, instead of the boundary conditions (5), one should use the conditions 


$$
\begin{aligned}
\sigma_{\mathrm{xz}} & =2 \mu \mathrm{u}_{\mathrm{xz}} \\
= & 0 \\
\sigma_{\mathrm{yz}} & =2 \mu \mathrm{u}_{\mathrm{yz}}=0 \\
\sigma_{\mathrm{zz}}=\lambda \mathrm{u}_{\mathrm{nm}}+2 \mu \mathrm{u}_{\mathrm{zz}} & =-\mathrm{T}_{\mathrm{zz}}(\rho, \mathrm{t})
\end{aligned}
$$

Solving the problem (4), (9)-(11) by the Green's function method and taking into account only generated Rayleigh waves one can derive the following expression for the vertical component of the surface vibration velocity spectrum:

$$
v_{z}(\rho, \Theta, \omega)=\left(\frac{2 \pi}{k_{R} \rho}\right)^{1 / 2} \frac{(-i \omega) k_{R} k_{T}^{2} v_{1}}{2 \pi \mu F^{\prime}\left(k_{R}\right)} T_{z z}(\omega) e^{-k_{R} \gamma \rho} e^{i k_{R} \rho-i 3 \pi / 4}
$$

Here $T_{z z}(\omega)$ represents the Fourier transform of $T_{z z}(0, t)$ which can be obtained from (9) and (10). In particular, it follows from (9) that

$$
z_{2}(\omega)=\frac{\omega_{1}^{2} Z_{1}(\omega)}{\sqrt{\left(\omega_{0}^{2}-\omega^{2}\right)^{2}+(2 \omega \alpha)^{2}}} \exp \left[-i \tan ^{-1}\left(\frac{2 \omega \alpha}{\omega_{0}^{2}-\omega^{2}}\right)\right]
$$

where $\omega_{0}=(\mathrm{K} / \mathrm{m})^{1 / 2}$ is the hop resonance frequency, $\omega_{1}=\left(\mathrm{K}_{1} / \mathrm{m}\right)^{1 / 2}$ is the tyre "jumping" resonant frequency, $\alpha=\mathrm{Q} / 2 \mathrm{~m}$ is a normalised damping coefficient, and $\mathrm{Z}_{1}(\omega)$ is the Fourier transform (spectrum) corresponding to the pit or bump profile.

Obviously, if the spectrum $Z_{1}(\omega)$ is wide enough, i.e., if the vehicle speed is comparatively high, then, according to (13), the axle vibrations at the hop resonance are effectively excited and noticeable generation of ground vibrations take place. One can show that in this case the force applied to the ground, $\mathrm{T}_{z z}\left(\omega_{0}\right)$, is proportional to $m \omega_{1}^{2}(\mathrm{hl} / v) \cos \left(\omega_{0} \mathrm{~L} / 2 v\right)$, where $\mathrm{h}$ is a characteristic height or depth of an obstacle and 1 is its characteristic length. Calculations of generated ground vibration amplitudes for typical values of $h$, 1 , and vehicle parameters show these amplitudes are generally higher than the amplitudes of vibrations generated by earlier considered accelerating and braking vehicles.

\section{CONCLUSIONS}

The generation of low-frequency ground vibrations by heavy lorries has been considered for both vehicles accelerating (decelerating) with a constant acceleration and vehicles travelling at constant speed on damaged or bumpy surfaces.

The amplitudes of ground vibration spectra generated by accelerating and braking lorries at medium and upper bands of the spectra are determined mainly by acceleration and are almost independent of the initial (final) vehicle speed. For low-frequency spectral bands, oscillations of ground vibration amplitudes versus both acceleration and initial speed may take place. These oscillations may be responsible for large statistical deviations of experimentally observed ground vibration levels.

Ground vibrations generated by lorries travelling on damaged or bumpy surfaces depend strongly on the relation between the width of the frequency spectrum corresponding to the profile of a pit or a bump and the axle-hop resonance frequency. For typical obstacle and vehicle parameters the amplitudes of ground vibrations due to pits or bumps are usually higher than the 
LOW-FREQUENCY RAYLEIGH WAVES

amplitudes of vibrations generated by accelerating and braking lorries.

\section{REFERENCES}

1. Watts, G.R., Vibration nuisance from road traffic - results of a 50 site survey. Transport Research Laboratory report LR1119 (Crowthorne 1984).

2. Beitin, K.I., Transactions of the ASME: J. Appl. Mech. No. 12, 819-826 (1969).

3. Krylov, V.V., Applied Acoustics, 44, 149-164 (1995).

4. Gutovski, T.G. and Dym, C.L., J. Sound and Vibr. 49, 179-193 (1976).

5. Hunt, H.E.M., J. Sound and Vibr. 144, 53-70 (1991). 